NOTICIAS Y COMENTARIOS 



\title{
LA PROBLEMÁTICA DEL MONTE MEDITERRÁNEO
}

\author{
Ricardo Vélez Muñoz \\ Sociedad Española de Ciencias Forestales \\ Cristina Montiel Molina \\ Dpto. de Análisis Geográfico Regional y Geografía Física \\ Universidad Complutense de Madrid
}

\section{INTRODUCCIÓN}

La Asociación Internacional Montes Mediterráneos (AIMM,www.aifm.org) desarrolló entre los años 1999 y 2001 un proyecto Interreg IIC Mediterráneo Occidental-Alpes Latinos titulado «Problemática del monte mediterráneo», donde participaron como socios las regiones de Provence-Alpes-Côte d'Azur y Campania, y el Parque del Vesubio, y en el que colaboró también la Comunidad Valenciana. La cooperación interregional en torno al tema se articuló a través de seis grupos de trabajo que abordaron los principales asuntos que afectan a los montes mediterráneos mediante seminarios internacionales coordinados por expertos en la materia y en los que participaron 198 especialistas de 12 países diferentes de la cuenca.

Los resultados de este proyecto han sido recientemente publicados por la AIMM como número extraordinario de la revista Forêt Méditerranéenne $\left(\mathrm{n}^{\mathrm{o}} 1\right.$ - 2002, www.foret-mediterraneenne.org). A continuación presentamos la versión española de las síntesis correspondientes a los seis grupos de trabajo, así como las Conclusiones de Valencia y la Declaración de Marsella sobre los montes mediterráneos, extraídas de dicha publicación.

\section{ESPECIFICIDADES ECOLÓGICAS DE LA CUENCA MEDITERRÁNEA}

\section{Síntesis del Seminario celebrado en Nápoles, marzo 2000, coordinado por Carlo Bifulco, Director del Parque Nacional del Vesubio, Italia}

El conocimiento de las características ecológicas de los montes mediterráneos permite examinar su gestión, los riesgos que les amenazan y las perspectivas de evolución. 


\section{- DEFINICIÓN DE LAS REGIONES MEDITERRÁNEAS}

Las regiones mediterráneas están situadas al oeste de los continentes, en latitudes subtropicales comprendidas, según los autores, entre los 30 y 40 grados o entre los 25 y 45 . Su clima se caracteriza por un fuerte déficit pluviométrico, durante la estación cálida, y por un invierno demasiado frío para que se puedan aprovechar las precipitaciones de esta estación. La biología de los organismos vegetales y animales está muy marcada por la limitación en la disponibilidad de agua.

Las regiones con bioclima mediterráneo son la cuenca mediterránea, parte de California (México y USA) y de Chile, la región del Cabo en Sudáfrica y las regiones de Melbourne y de Perth en Australia. Nos referiremos solamente a la Cuenca Mediterránea.

\section{- CARACTERÍSTICAS DE LA CUENCA MEDITERRÁNEA}

Las condiciones climáticas, a veces severas, del clima mediterráneo hacen que la recuperación de la vegetación sea, a veces, muy lenta e incluso irreversible.

La originalidad de este medio es la presencia del hombre desde la aparición del clima mediterráneo. Las consecuencias ecológicas de su acción son variadas.

En algunos casos la agricultura tradicional ha incrementado la diversidad paisajística, creando numerosos nichos ecológicos. En otros casos, la sobreexplotación del medio forestal o la industrialización ha favorecido la erosión e, incluso, la desertificación y es, probablemente, el origen de los cambios climáticos presentes y futuros.

\section{- EFECTOS DE LOS CAMBIOS CLIMÁTICOS}

El aumento de temperatura del planeta produciría los siguientes cambios:

- desplazamiento de las isotermas hacia el norte

- cambio del régimen de precipitaciones (disminución en las latitudes elevadas y aumento en las bajas)

- elevación del nivel del mar

Estos cambios pueden tener las consecuencias ecológicas siguientes:

- modificación de las zonas de distribución de las especies (desplazamiento hacia el norte y en altitud)

- modificación del metabolismo de las especies

- modificación de su comportamiento (sedentarización de los animales migratorios, modificación de la época de reproducción y de la fecundidad...)

En algunos casos estas modificaciones se han manifestado ya.

- ESPECIFICIDADES ECOLÓGICAS DE LOS ESPACIOS NATURALES Y FORESTALES MEDITERRÁNEOS

La Cuenca Mediterránea es reconocida por su riqueza y su especificidad florística y faunística. La heterogeneidad de los hábitats de repartición de las especies implica una varia- 
bilidad fenotípica elevada. Al considerar la diversidad del medio forestal, se debe tener en cuenta también las formaciones perisilváticas, incluyendo los matorrales, los pastizales y las estepas.

La biodiversidad de los espacios naturales y forestales mediterráneos varía según los taxones de un lugar a otro (por ejemplo, algunas regiones se caracterizan por su fuerte endemismo vegetal y la pobreza en avifauna) y es muy importante en:

- los espacios intermedios abiertos con matorrales, más que en los bosques.

— los espacios moderadamente alterados por el hombre.

La dinámica ecológica implica que la mayoría de los montes mediterráneos tienen baja producción leñosa en comparación con los bosques tropicales o boreales. En cambio es importante la producción no leñosa.

\section{- DINÁMICA ECOLÓGICA DE LOS MONTES MEDITERRÁNEOS Y LOS PRO- BLEMAS DE LA GESTIÓN SELVÍCOLA}

El norte del Mediterráneo (España, Francia, Italia y Grecia), debido al éxodo rural, presenta una dinámica espontánea de recolonización de las tierras agrícolas abandonadas por las especies forestales. El abandono de las superficies agrícolas da lugar a un incremento de la superficie forestal junto con la uniformización de los espacios forestales. La prioridad no es actualmente la reforestación, sino la gestión sostenible de estos ecosistemas en su dinámica presente.

Los montes del sur del Mediterráneo (Magreb y Oriente próximo) sufren una fuerte presión antrópica (pastoreo, recogida de leña, alimentación humana, plantas medicinales) que crea problemas de regeneración del arbolado y produce degradación de la fertilidad del suelo.

La problemática de los ecosistemas forestales se puede examinar considerando los usos dominantes. Se pueden distinguir:

- Espacios utilizados por una población rural de bajos ingresos. Su gestión se integra en el desarrollo rural.

- Espacios agrícolas abandonados. Hay que preguntarse si es necesaria la intervención humana en ellos. Parece útil identificar los espacios en los que la dinámica vegetal no se corresponde con los intereses de la sociedad humana y en los que el hombre debería intervenir para modificarla.

- Los espacios sometidos a la presión humana o a una demanda social fuerte (espacios periurbanos, parques). Los conflictos de uso con la utilización agropecuaria tradicional son frecuentes. En ellos aparece una problemática de protección, conservación y acogida del público.

- Los espacios destinados a la producción de madera.

\section{- LA PROBLEMÁTICA DE LA PROTECCIÓN DE LOS MONTES MEDITERRÁNEOS}

La dinámica ecológica muestra que los ecosistemas mediterráneos tienen gran capacidad de reacción ante las perturbaciones exteriores. Las acciones de protección de los medios naturales parecen indispensables en las zonas de alta densidad de población, a causa de la presión que ésta ejerce sobre el medio. 
¿Cómo se deben escoger los espacios que deben ser protegidos y con qué criterios? ¿Cuáles son las prioridades? La biodiversidad puede ser uno de estos criterios. Si se hace así, ¿qué aspecto de la biodiversidad debería ser prioritario?

En algunos casos se puede decidir la restauración o la conservación de estos espacios. Sin embargo, las series dinámicas de las plantas son difíciles de identificar, ya que la acción del hombre es antigua e intensa en toda la Cuenca. No siempre se puede identificar el tipo de selvicultura que se debe aplicar. Las medidas de conservación no permiten recrear siempre el estado que se desea.

- TEMAS DE ESTUDIO PARA CONSIDERAR EN EL PRIMER CONGRESO FORESTAL MEDITERRÁNEO

Se propone que el futuro Congreso estudie las especificidades ecológicas de los montes mediterráneos en los siguientes aspectos:

- La gestión forestal ligada a esas especificidades: ¿Qué instrumentos se deben utilizar para tenerlas en cuenta?

- Las consecuencias ecológicas de la acción del hombre: ¿Cuál ha sido y es su influencia? ¿Cómo prever el efecto de los cambios socioeconómicos sobre los montes?

- Efectos del cambio climático.

\section{CIVILIZACIÓN Y PATRIMONIO}

\section{Síntesis del Seminario celebrado en Aix-en-Provence, marzo 2001, coordinado por Andrée Corvol-Dessert, Directora de Investigación del CNRS de París (Francia)}

\section{- EL LUGAR DE LOS MONTES MEDITERRÁNEOS EN NUESTRAS SOCIEDADES}

Hasta el siglo XIX, en Francia y en Italia los montes mediterráneos estaban integrados en el sistema de producción agro-silvo-pastoral, en el cual desempeñaban una importante función económica. La densidad demográfica de los espacios rurales, que alcanzó en esta época sus máximos valores, supuso la sobreexplotación agrícola y la reducción progresiva de la superficie forestal.

En este contexto, surge una toma de conciencia acerca de la fragilidad de los medios forestales mediterráneos. El Estado intenta asegurar la protección de estos espacios, oponiéndose especialmente a los usos domésticos locales. Como resultado de ello, se multiplican los conflictos en relación a las regulaciones establecidas (respecto a la caza, al pastoreo...).

Los conflictos entre el Estado y los usuarios de los montes son la expresión de la voluntad de apropiación del espacio conformado por los montes mediterráneos. Además, ponen de manifiesto la aparición de una conciencia patrimonial de estos montes o de la naturaleza en general.

La problemática relativa a la sobreexplotación y a la conservación del medio se atempera con el éxodo rural. Este éxodo supone la expansión de los montes y la pérdida del conocimiento de las prácticas locales forestales y pastoriles. En el Magreb, la problemática es parecida, aunque el éxodo rural no se manifiesta hasta el siglo XX. Las poblaciones de 
los espacios forestales continuan siendo numerosas, aunque la presión se reduce como consecuencia de un uso menor de la leña.

El monte mediterráneo se «terciariza» progresivamente, perdiendo poco a poco su función primaria de producción e interesando cada vez más a los ciudadanos. Actualmente, una parte considerable de los montes está carente de gestión, ya que la sociedad rural se ha desarraigado del territorio y generalmente se desentiende del valor de estos espacios.

\section{- LA PERCEPCIÓN DEL VALOR DEL PATRIMONIO FORESTAL MEDITERRÁNEO}

El patrimonio es un bien que posee un valor económico, que se conserva y que se procura incrementar. La conservación del patrimonio forestal implica, por tanto, su gestión.

Las percepciones confrontadas que posee la sociedad acerca de sus bosques se ponen de manifiesto a través de las diferentes actitudes adoptadas ante la defensa de los montes contra los incendios.

Generalmente, la lucha se organiza contra los riesgos que amenazan a los bienes localizdos en los montes o en sus proximidad, más que contra los riesgos que amenazan al patrimonio forestal en sí mismo. Es prioritaria la protección de las personas y de los bienes. Existe, de esta manera, una apreciable diferencia entre la geografia forestal y la distribución espacial de los dispositivos de protección. La defensa del patrimonio forestal depende, por tanto, del valor que se le asigna en función de otros bienes (viviendas...).

El éxodo de las poblaciones rurales que empleaban el fuego en la gestión de estos espacios o para luchar contra los incendios, y la terciarización de los montes, han conducido a la criminalización del fuego, considerado como agente destructor del patrimonio forestal.

La percepción de este patrimonio ha evolucionado junto con la concepción del territorio y la representación social de los montes mediterráneos. Estos han sido sucesivamente valorados en función de los territorios que ocupaban (pastos, caza...), a continuación por su potencial de producción (madera, recolección de frutos...), y finalmente por su biodiversidad y valor paisajístico. Además, la percepción de este patrimonio depende también del contexto histórico y cultural de cada región y de cada grupo social. En un mismo territorio existe diferentes posibilidades según los grupos sociales que lo organicen, y un mismo paisaje posee un valor diferente en función del lugar y del momento.

En consecuencia, las orientaciones adoptadas para preservar este patrimonio no pueden ser universales. Es preciso aclarar previamente el modelo de bosques deseado. Actualmente, los criterios de elección de las políticas de protección que predominan son la diversidad paisajística y la biodiversidad. El valor de la biodiversidad se evalua asimismo en función de los equilibrios ecológicos y de las representaciones sociales.

\section{- LA PROTECCIÓN Y LA CONSERVACIÓN DEL PATRIMONIO FORESTAL MEDI- TERRÁNEO}

Proteger un espacio natural significa intervenir contra las presiones externas que supondrían su modificación. Pero la conservación, en el sentido de la fijación de un paisaje por ejemplo, puede inducir intervenciones radicalmente contrarias a la evolución.

Cada grupo social proyecta en el monte su percepción del mundo. El mito de la naturaleza virgen, sinónimo del paraíso terrenal, está fuertemente arraigado en la sociedad occidental. La protección del monte obliga, por tanto, a considerar la relación existente entre la sociedad y la naturaleza. 
¿El objetivo de la protección es acaso sustraer la naturaleza a la acción del hombre? ¿Se conseguiría realmente la protección de esta manera?

¿La protección exige inexorablemente una conservación inmobilista? ¿Es posible plantear la fijación del patrimonio forestal? Museificar una situación o un paisaje parece utópico, puesto que, como todo sistema biológico, el paisaje evoluciona y es imposible mantenerlo inalterado sin intervención externa.

\section{SOCIEDADES, TERRITORIOS, INSTITUCIONES}

\section{Síntesis del Seminario celebrado en Torre del Greco (Italia), diciembre 2000, coordinado por Americo Carvalho Mendes, Profesor de Economía en la Universidad Católica de Porto (Portugal)}

\section{- LAS ESPECIFICIDADES SOCIOECONÓMICAS Y TERRITORIALES DE LOS MONTES MEDITERRÁNEOS}

$\underline{\text { Las características de los montes mediterráneos }}$

\section{El valor de los montes mediterráneos}

Los montes y los espacios naturales terrestres mediterráneos se caracterizan por la escasa producción maderera y por la importancia que adquieren los productos no maderables y los servicios no comerciales (ocio, paisaje, turismo, marco de vida).

En las zonas dependientes de las actividades rurales, estos espacios están generalmente integrados en los sistemas de producción agrosilvopastoral. En la proximidad de las ciudades, el uso de los montes se terciariza. La evaluación de su valor es difícil, puesto que está cada vez menos ligado a una producción concreta, y más relacionado en cambio con la calidad de vida y con el atractivo turístico de la región.

Además, los montes mediterráneos constituyen un patrimonio cultural en todos los países de la cuenca mediterránea.

\section{La organización de las sociedades alrededor de los montes mediterráneos}

Los montes pertenecen a la colectividad o a propietarios particulares. Al norte del Mediterráneo, la mayor parte de la propiedad es privada y fragmentada, mientras que al sur y al este, los montes son mayoritariamente públicos (del Estado, comunidades y sociedades, etc.). Por otra parte, la categoría de propietario particular está en pleno proceso de transformación, debido al éxodo rural y a la llegada de propietarios no-rurales.

Los usuarios se dividen en dos grupos, según sus actividades impliquen una dinámica forestal (selvicultores, población local de economía basada en los recursos forestales, ganaderos, cazadores...) o, por el contrario, aspiren a la estabilización de los montes y del paisaje para el desarrollo de actividades de ocio.

La diversidad de los montes mediterráneos obedece tanto a los sistemas de gestión como a los usos de la población y a las herencias naturales, culturales e históricas asociadas a cada territorio. 
$\underline{\text { Las problemáticas actuales de los montes mediterráneos }}$

Los cambios de relaciones de producción, de economía y en el territorio, especialmente patentes al norte del Mediterráneo, han inducido la evolución de las expectativas de la sociedad frente a los montes. Las poblaciones son cada vez más urbanas y la demanda de turismo aumenta. En consecuencia, el monte se valora como un espacio natural de consumo, más que como una zona de producción o de abastecimiento.

Estos cambios han alterado igualmente los equilibrios rurales existentes. Buena parte de los montes está al margen de cualquier tipo de gestión, debido al desarraigo territorial de la población local.

Se observa un desencuentro entre los propietarios, que apenas explotan sus montes debido a su escasa rentabilidad, y los usuarios, que reivindican su protección y mantenimiento. La demanda social hacia los espacios naturales no siempre es compatible con ciertos intereses económicos y con los retos del desarrollo.

Los problemas forestales de la cuenca mediterránea dependen del contexto socioeconómico de cada país. Se trata de montes complejos, multifuncionales y con múltiples usuarios, que no pueden ser gestionados de acuerdo con los modelos norte-europeos estandarizados. Es conveniente, en cada caso, valorar los usos de que son objeto para plantear a continuación modelos de aprovechamiento sostenible.

\section{- EL CONGRESO MEDITERRÁNEO DE LOS BOSQUES Y DE LOS ESPACIOS NATURALES TERRESTRES}

\section{Los principios y objetivos del Congreso}

El modelo de ordenación del territorio mediterráneo difiere del modelo de especialización espacial característico de la zona situada al norte de la región mediterránea (donde están diferenciadas las zonas de producción industrial y agrícola, los espacios urbanos y los espacios naturales).

El objetivo de este Primer Congreso Mediterráneo de los Bosques y de los Espacios Naturales Terrestres es contribuir a la definición de un modelo de gestión de los montes mediterráneos adaptado a sus especificidades, a sus nuevas oportunidades y a las dificultades a las que se enfrenta, partiendo del conjunto de sus potencialidades. Para ello, es fundamental el reconocimiento de las especificidades de los montes mediterráneos y, sobre todo, que sus problemáticas sean incorporadas a los congresos forestales internacionales, de manera que estos espacios se integren adecuadamente en las políticas de desarrollo y de ordenación del territorio.

Este evento será una ocasión oportuna para:

- mostrar diferentes ejemplos de gestión de los espacios forestales mediterráneos,

- reforzar la cooperación entre las instancias de decisión política, entre las ONGs (asociaciones de proprietarios forestales, asociaciones ecologistas, asociaciones de desarrollo local...) y entre los investigadores y los profesionales.

\section{$\underline{\text { La estructura del Congreso }}$}

El Congreso no debe ser una manifestación puntual, sino una etapa en un proceso continuo de cooperación. Para que sea un lugar de intercambios abierto a todas las componen- 
tes de la sociedad, y que esos intercambios faciliten una construcción colectiva eficaz, el Congreso debe integrar:

— un apartado político de propuesta de estrategias y medidas necesarias para el desarrollo de los montes mediterráneos,

- un apartado profesional y científico de discusiones temáticas, bajo la fórmula de talleres, sobre las problemáticas fundamentales,

- un apartado de intercambio de experiencias de desarrollo que agrupe las exposiciones y comunicaciones sobre proyectos territoriales.

\section{El apartado político}

El aspecto político del Congreso consistirá en debatir una resolución que constituya la base de un Acuerdo Internacional sobre los bosques y los espacios naturales terrestres mediterráneos y que será presentada a las diferentes estructuras institucionales.

Esta resolución debe incluir las siguientes cuestiones:

- la importancia de la perspectiva territorial,

- la necesidad de formular un modelo mediterráneo de gestión y de ordenación del territorio,

- la necesidad de abordar el monte mediterráneo en relación con la protección de los recursos naturales, con la sociedad y con el medioambiente.

Esta propuesta se orienta hacia la ampliación de la participación en los procesos de decisión, sin usurpar las competencias de las instituciones gubernamentales.

\section{El apartado profesional y científico}

Los aspectos socioeconómicos y territoriales serán examinados en el Primer Congreso Mediterráneo de los Bosques y de los Espacios Naturales Terrestres, planteando desde diferentes ángulos la diversidad y la multifuncionalidad de los montes mediterráneos. Los cinco temas de trabajo que se proponen son:

- Inventario de los bienes y servicios, comerciales y no comerciales, que ofrecen los montes mediterráneos, y demostración de su especificidad respecto de otros tipos de montes

- Análisis de los enfoques territoriales (que difieren de los enfoques sectoriales y de los modelos de especialización territorial) incorporando las diferentes dimensiones espaciales a la investigación científica.

- Análisis de las dinámicas naturales y socioeconómicas que se encuentran en el origen de los riesgos que amenazan a los montes mediterráneos (incendios, cambios climáticos, presión urbana, usos agrícolas, desertificación; así como de las políticas que hacen aumentar o mitigan los efectos de dichos riesgos.

- Valoración técnica y económica de los proyectos-piloto territoriales que ofrecen nuevas posibilidades de desarrollo en el contexto de los cambios sociales contemporáneos. 
$\underline{\text { Los proyectos de sitios-piloto }}$

A la par que el Congreso, está previsto el funcionamiento de una serie de experienciaspiloto donde, a través de los intercambios técnicos, se llevará a cabo la reflexión acerca de los problemas forestales comunes. Los temas de cooperación propuestos son los siguientes:

— La gestión de áreas afectadas por limitaciones ambientales y las técnicas de «ingeniería biológica».

- Los problemas de la propiedad forestal.

- La gestión de espacios afectados por el riesgo de incendios forestales (análisis de los factores socioeconómicos coadyuvantes de los incendios).

- El problema del abandono de tierras agrícolas y la pérdida de gestión forestal.

- El uso terciario del monte mediterráneo.

- Los problemas ligados al minifundismo en los espacios forestales.

- Seguimiento de la evolución de los territorios.

\section{RIESGOS PARA LOS MONTES MEDITERRÁNEOS A COMIENZOS DEL SIGLO XXI}

\section{Síntesis del Seminario celebrado en Valencia, junio 2001, coordinado por Ricardo Vélez, Ministerio de Medio Ambiente, Madrid (España).}

En el marco del Proyecto INTERREG II C «Problemática del monte mediterráneo», al que están asociadas las regiones Provenza-Alpes-Costa Azul (Francia) y Campania (Italia), el Grupo de Expertos sobre Riesgos, de la Asociación Internacional Montes Mediterráneos (AIFM), reunido en Valencia por invitación de la Consejería de Medio Ambiente, de la Generalitat Valenciana, durante los días 8 y 9 de junio de 2001, ha examinado la situación de los montes de la Cuenca Mediterránea, llegando a las siguientes conclusiones, denominadas CONCLUSIONES DE VALENCIA:

1. Los montes mediterráneos son el conjunto de espacios naturales terrestres de bioclima mediterráneo y comprenden desde los territorios más degradados por la erosión hasta los bosques más bellos.

Estos montes están sometidos a una serie de riesgos, tanto de origen natural como humano, que pueden amenazar sus funciones protectoras, productoras, sociales y culturales, necesarias para el adecuado desarrollo de la vida humana en esta región del mundo.

2. Algunos riesgos, como el de los incendios forestales, han evolucionado, agravándose en las últimas décadas, influyendo negativamente en los demás problemas, como la erosión, la extensión de plagas y enfermedades, la disminución de los recursos de agua y el avance de la urbanización.

Su interacción con fenómenos naturales, como la sequía, incrementa la vulnerabilidad de los montes y pone en peligro la seguridad de las personas, haciendo necesaria la aplicación de medidas preventivas que eviten los efectos acumulativos de los diferentes riesgos. 
3. En la evolución de los riesgos influyen factores socioeconómicos, como el abandono de tierras en países del norte del Mediterráneo o la presión antrópica sobre el medio forestal en los países del sur de la Cuenca o la distancia cultural de la población urbana respecto de la rural.

4. Los riesgos a los que están sometidos los montes mediterráneos no son bien comprendidos por otros países situados fuera de la Cuenca, lo que produce bien efectos negativos de determinadas políticas diseñadas en organizaciones internacionales, bien desatención de las mismas.

En particular se señalan los efectos en situaciones particulares de las subvenciones de la Unión Europea para la ganadería de ovejas y cabras en el incremento del riesgo de incendios forestales y de erosión por falta de una correcta planificación y cooperación entre la selvicultura y la ganadería.

Asimismo la FAO ha disminuido notablemente la actividad que desarrollaba a través de su Comité Silva Mediterránea.

Esta situación contrasta con la necesidad de acciones contra la desertificación, riesgo final que afecta a extensas zonas de la Cuenca Mediterránea.

5. Se considera necesario hacer comprender a la sociedad que el monte mediterráneo juega un papel básico en la calidad ambiental de la Cuenca. Parece indispensable concebir y poner en práctica una política forestal específicamente mediterránea.

Para ello es preciso desarrollar una visión amplia de la gestión forestal, alrededor del núcleo central de la selvicultura, y en coordinación con las políticas de ordenación del territorio con objeto de:

- Hacer que el monte sea deseable y útil para toda la población y compatible con sus necesidades reales.

- Asegurar una gestión correcta, basada en el conocimiento científico y técnico, considerando la interrelación entre los riesgos de incendios, plagas y enfermedades, erosión e inundaciones y pérdida de biodiversidad.

- Reunir a todos los que tienen interés en el monte mediterráneo para que expresen sus ideas sobre las políticas que deben desarrollarse y cooperen en su aplicación. Esto incluye a los propietarios forestales públicos y privados, a los forestales profesionales, a los científicos, a los servicios contra incendios, a las autoridades locales, a los medios de comunicación, a las ONG y a los organismos que gestionan los espacios protegidos.

6. Se considera que la prevención y control de los riesgos que amenazan a los montes no pueden ser realizados sin la implicación de sus propietarios, mayoritariamente privados al norte del Mediterráneo, y de la población que los utiliza, al sur de la Cuenca.

Para ello se hace preciso promover eficazmente figuras asociativas, que permitan la gestión sostenible de los montes, tal como ha venido siendo reconocido en distintos foros internacionales, entre otros las cumbres ministeriales de Helsinki y Lisboa.

7. Se considera indispensable el seguimiento de los daños provocados por los distintos riesgos para el conjunto de la Cuenca Mediterránea, con objeto de conocer su evolución y sus magnitudes para la previsión de los problemas a corto y medio plazo, utilizando cartografía de factores de riesgo en general.

En particular debería apoyarse la consolidación de la base de datos descentralizada sobre incendios forestales iniciada en los años 90 por el Comité FAO/Silva Mediterránea. 
Asimismo se considera necesaria la elaboración y divulgación de mapas de peligro de incendios forestales durante la época de mayor riesgo cubriendo todos los países de la Cuenca Mediterránea.

Además se considera necesaria la extensión de la Red de Seguimiento del Estado Sanitario de los Bosques, que actualmente cubre gran parte de Europa, tanto al Norte de Africa como a la zona oriental del Mediterráneo.

Para todo ello se considera necesario dirigirse a la Comisión Europea para mostrar el interés por la renovación y fortalecimiento de sus Reglamentos para la protección de los bosques contra los incendios y contra la contaminación.

8. Se considera necesario diseñar una estrategia mediterránea de ayuda mutua en caso de situación de peligro extremo de incendios forestales, o de plagas extraordinarias, como la langosta, mediante acuerdos bilaterales y multilaterales entre Estados, que permitan trasladar medios técnicos y de intervención desde las zonas donde estén disponibles a aquéllas en que la situación sea más grave.

9. Se considera necesario estudiar la peligrosidad de otros factores como agentes de alteración del ambiente forestal mediterráneo, tal como el desarrollo de las especies invasoras tanto de fauna como de flora.

10. Como recomendación final, se considera necesario el tratamiento de los riesgos en el monte mediterráneo desde un enfoque preventivo y de acuerdo con el modelo actual y futuro que imponen las dinámicas socioeconómica y forestal.

Para ello se propone continuar los trabajos iniciados con el Proyecto «Problemática del monte mediterráneo», en particular en el marco del Programa INTERREG III, y que la Asociación Internacional Monte Mediterráneo (AIMM) mantenga su acción a favor del intercambio de información.

En este sentido los participantes recomiendan especialmente que sea organizado en un próximo futuro el Primer Congreso Mediterráneo de Montes y Espacios Naturales Terrestres.

\section{FORMACIÓN, INVESTIGACIÓN, SENSIBILIZACIÓN}

\section{Síntesis del Seminario celebrado en Marsella, Francia, mayo 2001, coordinado por Michel Bariteau, INRA-Aviñón, Francia}

\section{- LA INVESTIGACIÓN Y LA FORMACIÓN SOBRE LOS MONTES MEDITERRÁNEOS}

\section{Dificultades para su desarrollo}

Las dificultades que encuentran la investigación y la formación sobre los montes mediterráneos proceden del desinterés por estos ecosistemas a nivel internacional, así como de la falta de percepción de su valor y de la inadecuación de los conceptos aplicados frecuentemente para gestionarlos.

Los problemas forestales de la Cuenca Mediterránea dependen del contexto socioeconómico y de la historia de las regiones que la componen. En la década pasada se han ido poniendo de relieve estas dimensiones, pero no es fácil integrarlas en los programas de investigación y formación. 
El monte mediterráneo es multifuncional y multiforme y, por tanto, con múltiples usuarios. Su gestión no puede basarse en los modelos aplicables para la producción de madera, ya que están localizados en territorios intermedios entre zonas urbanas y agrícolas.

Se necesitan, por ello, modelos específicos adaptados a la situación mediterránea.

El conocimiento del monte mediterráneo como entidad coherente facilitaría las relaciones entre investigadores y gestores. Las características mediterráneas podrían conceptualizarse cartográficamente según sus diferentes definiciones.

\section{Adecuación de la investigación y de la formación a las demandas sociales}

La utilidad social de la investigación debe ser uno de sus objetivos. Para alcanzarla es precisa la concertación entre investigadores, gestores y usuarios, mediante transferencia de resultados para definir las prioridades de investigación.

La investigación debe estar relacionada con las necesidades regionales. Sin embargo, su financiación, al Norte del Mediterráneo, depende de los programas europeos, cuyas prioridades, a menudo, no coinciden con las problemáticas regionales mediterráneas. Es necesaria una política mediterránea de investigación que, actualmente, no está definida con claridad.

La enseñanza sobre espacios naturales y forestales mediterráneos se orienta frecuentemente a la gestión ambiental y a las nuevas tecnologías.

Los temas forestales se analizan esencialmente por los problemas de incendios e impactos ambientales, sin desarrollar una gestión forestal específica ni la dimensión socioeconómica.

Para adaptarse a esta situación, el sector forestal debe abrirse a la enseñanza típicamente «no forestal» y favorecer la difusión de la enseñanza selvícola en otros campos.

\section{Estructuración de la investigación sobre los montes mediterráneos}

La falta de coordinación en la elaboración de programas de formación e investigación produce redundancia en algunos temas y lagunas en otros. La armonización de programas y la promoción de intercambios es deseable.

La dispersión de responsabilidades y las dificultades de coordinación entre investigadores crean problemas para la financiación de los trabajos. La reestructuración de las instituciones aumentaría las probabilidades de elegibilidad de los proyectos de investigación.

\section{- LA COMUNICACIÓN SOBRE LOS MONTES MEDITERRÁNEOS}

La sensibilización sobre los montes mediterráneos precisa identificar sus destinatarios, así como los conceptos que deben transmitirse, de lo que se derivarán la naturaleza y contenido de los mensajes para lograr una eficacia adecuada.

\section{$\underline{\text { Objetivos de la sensibilización }}$}

Existe un desinterés general de la población por sus montes. Por tanto el primer objetivo es luchar contra la indiferencia para que sus necesidades sean tenidas en cuenta en los planes de gestión a nivel nacional e internacional. Una vía para ello sería el futuro Primer Congreso Mediterráneo sobre Montes y Espacios Naturales Terrestres, cuya organización está estudiando la AIMM. 


\section{Destinatarios}

Son de tres tipos: Los dirigentes y gestores, los ciudadanos que utilizan el monte y los propietarios forestales. Los primeros son los principales destinatarios de estos mensajes. Es preciso, por ello, identificarlos de modo explícito.

La Comisión Mediterránea para el Desarrollo Sostenible (CMDD) podría constituir un objetivo interesante.

\section{Visión del monte mediterráneo por los destinatarios}

Además es preciso identificar como ven los destinatarios el monte mediterráneo, para que puedan llegar a entender los mensajes, que deben adaptarse a ellos.

El monte es considerado, según los grupos sociales, como:

- fuente de recursos o medio natural

- monte ordenado o bosque, lo que plantea la cuestión de si es necesaria la gestión forestal.

— monte densamente poblado o monte integrado con otros medios en grado variable.

Los montes de cada región se caracterizan por una función o interés específicos, que dependen del contexto socioeconómico local, que es necesario identificar para adaptar a él los mensajes.

\section{Mensajes que se deben difundir}

Los mensajes sobre el monte mediterráneo difieren según las dinámicas de la vegetación y las organizaciones sociales. Los intereses de la población, propietarios o usuarios, y de la propiedad, privada o domanial, no son los mismos al Norte y al Sur del Mediterráneo. Sin embargo hay coincidencia en la necesidad de sensibilización de todos los destinatarios.

Hay varios temas para transmitir:

- el de la protección del medio forestal, insistiendo en su fragilidad y en los riesgos que le amenazan. Al Norte del Mediterráneo la protección contra el fuego es un tema aglutinante.

- el de la promoción del monte, insistiendo en la biodiversidad, la historia y su papel en las sucesivas civilizaciones. El monte mediterráneo es atípico, pero posee valores que es preciso aprovechar: su diversidad, la estructura en mosaico, la población, la contribución a dichas civilizaciones, la luminosidad, la riqueza en plantas aromáticas, los paisajes, etc.

La complejidad de los montes mediterráneos obliga a variar los mensajes en función de las situaciones:

- Para los dirigentes y gestores se podría insistir en la promoción del monte mediterráneo para desarrollar políticas más amplias que, actualmente, casi se limitan a la lucha contra los incendios.

- Para los usuarios se podría insistir en la fragilidad del monte mediterráneo y en su papel contra la desertificación y en la regulación de los recursos hídricos. 
- Para los propietarios forestales se podría insistir en las posibilidades de mejorar la productividad mediante mejoras técnicas de la gestión.

\section{Canales de comunicación}

El monte no es tema de información, salvo cuando arde o cuando sufre las consecuencias de una catástrofe natural, como los vendavales. Sería por ello útil asociar otros temas como el arte, la literatura, la cultura, la gastronomía o el cambio climático, la retención de carbono, etc. Sin embargo hay una pregunta importante: ¿Cómo conciliar la eficacia mediática y la verdad científica?

Entre los canales de comunicación, la televisión no parece adecuada, ya que el monte no es un tema de actualidad. La radio permite gran difusión de los mensajes, especialmente entre los usuarios del monte al Sur del Mediterráneo. Los manuales de divulgación se pueden dirigir a los propietarios y al público en general.

La utilización de certificaciones de sostenibilidad puede ser otro cauce para influir en los dirigentes que diseñan las políticas europeas que deben considerar la especificidad mediterránea. La comunicación dirigida al público en general es también un medio indirecto de sensibilizar a los dirigentes.

\section{COOPERACIÓN}

\section{Síntesis del Seminario celebrado en Marsella (Francia), noviembre 2001, coordinado por Jean Bonnier, Secretario Ejecutivo de la Asociación Internacional Montes Medite- rráneos}

\section{- LOS OBJETIVOS DE LA COOPERACIÓN SOBRE LOS MONTES MEDITERRÁNEOS}

El reconocimiento de la especificidad ecológica, social, económica, cultural e institucional de los espacios naturales y forestales mediterráneos es fundamental para abordar cualquier política de cooperación. La consideración de esta realidad en los procesos de decisión favorecerá una mejor adecuación de las acciones que se emprendan a las necesidades de los agentes territoriales del ámbito mediterráneo.

La definición de políticas forestales adecuadas a las estrategias de desarrollo territorial y a las necesidades sociales requiere la implicación de todos los agentes sociales afectados. Los proyectos de cooperación deberían surgir de las necesidades de los agentes locales, y ser planteados a través de los problemas locales existentes.

El principal objetivo de la cooperación sobre los montes mediterráneos es llegar a definir modelos de ordenación del territorio y de ordenación de estos espacios que respondan a las necesidades de las sociedades mediterráneas y a la especificidad de estos medios.

\section{- LAS ÁREAS PRIORITARIAS DE COOPERACIÓN}

\section{$\underline{\text { El conocimiento de los montes mediterráneos }}$}

Pese a la existencia de numerosos estudios, aunque poco coordinados y por tanto no acumulativos ni comparables entre sí, se percibe la necesidad de conocer el estado de la cues- 
tión y, a continuación, profundizar en el conocimiento de la biodiversidad y de la evolución futura de estos medios. Estos estudios deben partir de las circunstancias locales (erosión, desertificación, estado fitosanitario de los montes, contaminación...) así como del contexto social (estructura de la propiedad, demandas sociales, valor económico de los montes para la población rural...).

Es preciso igualmente profundizar en el conocimiento y en la difusión de las prácticas tradicionales, así como en la reciente evolución de los conflictos de usos.

Deben ser evaluados la rentabilidad social y el valor económico total de los montes mediterráneos, sobre todo considerando la escasa aportación de los productos comerciales a la renta de las sociedades locales.

La cooperación en todos estos ámbitos permitiría evitar lagunas y redundancias y favorecería igualmente la difusión de las experiencias adquiridas.

Existen ya redes de investigación, de ámbito mediterráneo o de mayor escala. Sin embargo, no existe un foro de discusión de las prioridades en materia de investigación. Además, con frecuecia se echa en falta la multidisciplinariedad en los proyectos de investigación.

\section{La formación}

El interés social de los montes mediterráneos justifica la necesidad de desarrollar la multidisciplinariedad en la enseñanza forestal y de extender la formación forestal a estudiantes de otras ramas, desde los cursos elementales y de forma continuada.

Por otra parte, sería positiva la coordinación en la programación de las enseñanzas impartidas por diferentes organismos de la cuenca mediterránea.

\section{$\underline{\text { La gestión de los espacios forestales }}$}

En materia de cooperación sobre el tema de la gestión de los espacios forestales, las acciones se han concentrado en:

- las técnicas de gestión de las formaciones originadas por regeneración espontánea de la vegetación, la introducción de especies exóticas, las repoblaciones y la «ingeniería biológica»,

- la promoción de iniciativas particulares relativas a la multifuncionalidad social y a la puesta en valor de los montes.

Resulta fundamental la puesta en común y el intercambio entre los diferentes propietarios forestales (públicos y privados), así como la incorporación de nuevos interlocutores, derivados de los procesos de descentralización y de la aparición de ONGs, para formular políticas de gestión consensuadas y mejor adaptadas a las especificidades mediterráneas.

Es necesario el desarrollo de la cooperación en la lucha contra incendios forestales y contra los riesgos fitosanitarios (dispositivos y material de lucha, puesta en común de información, colaboración en el desarrollo de nuevos métodos). Las redes actuales están inadaptadas a la región o son poco activas. 


\section{$\underline{\text { La comunicación }}$}

Los montes mediterráneos apenas son objeto de consideración en la puesta en valor y ordenación del territorio. Su valor económico se ve a menudo eclipsado por el problema de los incendios.

Es necesaria la comunicación a partir de la realidad social, territorial y técnica de los montes mediterráneos para vencer el desinterés de las sociedades locales por sus montes, para lograr una aproximación entre los selvicultores y los ecologistas y para hacer valer el lugar que corresponde a los propietarios y a los gestores forestales.

\section{- LAS MODALIDADES DE COOPERACIÓN}

La evaluación de las modalidades de cooperación actualmente existentes en las regiones mediterráneas conduce a la identificación de una serie de lagunas y barreras, así como sus causas.

Aparte de Silva Mediterránea, apenas existe redes de cooperación en torno a los montes mediterráneos. En ausencia de un foro de relaciones explícitamente designado, la Asociación Internacional Montes Mediterráneos está capacitada para desempeñar, al menos de forma parcial, esta función y contribuir a la gestación de nuevos modelos forestales mediterráneos.

Se propone la experimentación de un modelo de cooperación «conectado con el territorio», a través de acciones concretas en el seno de sitios-piloto. Los socios de la cooperación seleccionarán los sitios-piloto (cuenca vertiente, parque natural, comarcas, mancomunidades, etc...) que serán la sede de intercambios personales y técnicos. Estos intercambios permitirán ensayar fórmulas de trasferencia de métodos de procedimientos. Los avances realizados serán de esta manera capitalizados y puestos en conocimiento de los demás socios y agentes interesados, especialmente a través del Primer Congreso Mediterráneo de los Bosques y de los Espacios Naturales Terrestres. De esta manera se construirá una cultura forestal mediterránea compartida.

La financiación de esta cooperación es un aspecto básico al que se deberá igualmente prestar atención.

\section{DECLARACIÓN DE MARSELLA SOBRE LOS MONTES MEDITERRÁNEOS}

\section{Conclusiones del Seminario de Clausura celebrado en Marsella (Francia), noviembre 2001}

Los asistentes al Seminario celebrado en Marsella el día 6 de noviembre de 2001 para aprobar las conclusiones del Proyecto «Problemática del monte mediterráneo», desarrollado por la Asociación Internacional Montes Mediterráneos (AIMM) en el marco del programa de interés comunitario INTERREG IIC Mediterráneo Occidental-Alpes Latinos, con la participación del Estado francés, de las regiones de Provenza-Alpes-Costa Azul y Campania y del Parque Nacional del Vesubio, y la colaboración de la Comunidad Valenciana, comparten los siguientes planteamientos:

1. Los bosques y los espacios naturales terrestres mediterráneos (en adelante, los montes mediterráneos) constituyen la trama heredada del pasado sobre la cual las socie- 
dades locales han organizado el territorio. Campos, infraestructuras de producción y de transportes, núcleos de población y zonas de ocio, todo el espacio humanizado, se inscribe en el contexto constituido por los montes y gestionado por los propietarios forestales públicos y privados.

2. Las especificidades y riquezas ecológicas, forestales y socioeconómicas de la región mediterránea son insuficientemente consideradas en los foros europeos e internacionales donde se decide el porvenir de los montes.

3. Tanto en el norte como en el sur de la cuenca mediterránea, a pesar de existir diferentes contextos socioeconómicos, se observa por igual los problemas que encuentran los silvicultores y los expertos en medioambiente para interconectar sus conocimientos.

4. Al mismo tiempo, la tendencia general hacia la descentralización que afecta prácticamente a todos los países, ha provocado la proliferación de los lugares donde se decide la gestión del territorio y la asignación de recursos públicos a los montes mediterráneos, sin garantizar en todos los casos la coordinación.

5. Existe el riesgo de que el desconocimiento de la especifidad forestal mediterránea, unido a la fragmentación de enfoques, impidan a los gestores la consideración de dichas particularidades.

6. Deseamos y proponemos que las reflexiones, los intercambios y los trabajos iniciados en el marco de este programa INTERREG IIC puedan ser continuados:

- mediante un acercamiento entre los diferentes proyectos que afectan más o menos directamente a los espacios forestales mediterráneos, con objeto de enriquecer su gestión desde perspectivas diferentes;

- mediante la continuidad de las actividades y de las redes de socios vinculadas al proyecto «Problemática del monte mediterráneo»,

- mediante la ejecución de acciones de cooperación a través del proyecto «Organización de redes y de acciones de cooperación sobre el monte mediterráneo», en el marco del proyecto INTERREG IIIB, MEDOC y SUDOESTE, y en otros programas europeos (ARCHIMED, MEDA);

- mediante la puesta en marcha del proyecto del Primer Congreso Mediterráneo sobre los montes y los espacios naturales terrestres.

7. Las bases del Primer Congreso Mediterráneo sobre los Montes y los Espacios Naturales Terrestres han sido ampliamente establecidas durante el desarrollo del «Problemática del monte mediterráneo», y permitirán su celebración a partir del año 2003. La organización del Segundo Congreso, el año 2006, en otra sede, asegurará la consolidación y la utilidad de las redes organizadas para favorecer la consideración e integración del monte mediterráneo en los planes de ordenación y gestión sostenible del territorio.

8. La AIMM reúne las condiciones más idóneas para coordinar y dinamizar estas redes, así como para la ejecución de dichos proyectos internacionales, necesariamente colectivos e interdisciplinares. 
